\title{
Efficacy of exogenous phytase supplementation on production performance of commercial broiler
}

\section{Parvin E, MA Khatun, T Yeasmin and MM Sharmin*}

Department of Dairy and Poultry Science, Hajee Mohammad Danesh Science and Technology University, Dinajpur, Bangladesh

\begin{abstract}
An experiment was conducted to investigate the effect of supplementing of exogenous phytase enzyme on productivity of commercial broilers. Four strains of commercial broiler such as Fast feather, Arbor acres, Cobb-500 and Hubbard classic each having 78 numbers were reared for a period of 5 weeks. A total number of 312 day old broiler chicks were distributed into two dietary treatments i.e. basal diet (Control- $\mathrm{T}_{0}$ ) and basal diet supplemented with phytase enzyme $1 \mathrm{~g} / \mathrm{kg}$ feed (supplemented- $T_{1}$ ). The results indicated that broilers fed on diets supplemented with phytase have gained more body weight when compared with control group $(P<0.05)$. The first and second highest body weights were recorded for the strains Cobb-500 and First feather broilers, respectively, which feed on diet containing phytase. Feed consumption varied significantly between the broilers on different dietary regimes. Significant differences $(P<0.01)$ were found in feed conversion ratios among the birds fed on diet with phytase enzyme. The lowest feed conversion ratio was in Cobb-500 fed on phytase diet where as the highest was in Fast Feather provided with only basal diet during the $5^{\text {th }}$ week of age. Average feed conversion ratios were 1.73 and 1.86 for the supplemented and control group respectively. Use of phytase as feed additive in the diet resulted in high profitability/ broiler. Net profit per live broiler was the also higher in treatment group compared with control groups of birds. The highest net profit per live broiler was observed in Arbor Across strains fed on phytase diet. The result of the present study suggests that the addition of dietary phytase enzyme in the diet of broiler may increase the production performance and reduce the cost of production.
\end{abstract}

Key words: phytase, feed conversion ratio, broiler performance

Bangladesh Animal Husbandry Association. All rights reserved.

Bang. J. Anim. Sci. 2016. 45 (3): 31-39

\section{Introduction}

In commercial poultry ration, nutrients are fortified through accumulation of different feed ingredients furnished with necessary micro nutrients. Protein costs incur about 45 percent of the total feed cost. The daily requirements of dietary protein are furnished from different animal and plant sources. But the plant sources sometimes become harmful to the poultry as because plant sources contain some anti nutritional factors like phytate phosphorus, trypsin inhibitors, non-starch polysaccharides (NSP), oligosacchariedes and lectines. Phytate is the principal storage form of phosphorus in many plant tissues, especially bran and seeds. Phytate has been shown to block the absorption of not only phosphorus but also other minerals, particularly calcium, magnesium, iron, and zinc.
Phytate has also been shown to negatively affect the absorption of lipids and proteins. Approximate 60 to $80 \%$ of total phosphorus in plant feedstuffs is phytic Phosphorus (NRC, 1994). The bioavailability of phytic $P$ for monogastrics such as pigs and birds were rather low, owing to the lack of endophytase in the gastrointestinal tract. In order to make $\mathrm{P}$ available to broiler chicks, Phosphorous from plant sources must be hydrolyzed, with phytase as a catalyst, to inositols and inorganic phosphates which are readily absorbed in digestive tract.

Research has shown that the performance of broilers fed with different levels of inclusion of phytase and low levels of available $P$ in the diets can improve with the addition of the enzyme (Dilger et al., 2004; Fukayama et al., 2008). The supplementation of poultry diets with

\section{*Corresponding author: sharminah101_bau@yahoo.com}


exogenous enzymes to enhance their performance is not a new concept. A phytase (myo-inositol hexakisphosphate phosphohydrolase) is any type of phosphatase enzyme that catalyzes the hydrolysis of phytic acid (myo-inositol hexakisphosphate). The positive effect of phytase can be expressed through better appetite, low feed conversion ratio, high carcass quality and quick digestibility. Also, adding phytase decreases the amount of total and soluble phosphorus in the litter, which has positive effect on the environment when poultry litter is used as fertilizer. Phytase may reduce the cost of the diet by reducing the amount of soybean meal, fat and crystalline amino acids that must be added. Because of the positive effect of phytase on trace mineral utilization commercial use may lead to minimal use of trace minerals in diets where phytase is added. It has been shown that removing the trace mineral premix from poultry diets from hatch to 42 days has no effect on growth performance, but it does have negative effects on bone growth. This negative effect was not overcome by the addition of phytase, indicating that phytase may not be able to replace the trace mineral premix in diets of broilers. Therefore, the present research work was designed with the following objective:

i) To investigate the effect of exogenous phytase enzyme on productivity of different strains of commercial broilers.

ii) To evaluate the economic benefit of rearing broiler feed on diet containing phytase enzyme.

\section{Materials and Methods}

\section{Statement of the experiment}

The experiment was conducted at the open sided poultry shed located in the compound of the Dept. of Dairy and Poultry Science in Hajee Mohammad Danesh Science and Technology University, Dinajpur. There were four strains of commercial broiler such as Fast feather, Arbor acres, Cobb-500 and Hubbard classic each having 78 number used for the experiment for a period of 5 weeks. A total number of 312 day-old broiler chicks were used to find out the effect of exogenous phytase enzyme on the production performance of broiler.

\section{Layout of the experiment}

The chicks were randomly distributed to eight dietary treatment groups $\left(\mathrm{T}_{0} \mathrm{~F}, \mathrm{~T}_{0} \mathrm{~A}, \mathrm{~T}_{0} \mathrm{C}, \mathrm{T}_{0} \mathrm{H}, \mathrm{T}_{1} \mathrm{~F}\right.$, $T_{1} A, T_{1} C, T_{1} H$ ) having three replications in each treatment. The chicks were reared in separate pens according to treatments and replications, each dietary treatment group consisted of 39 chicks and numbers of chicks in each replication was 13. The layout of the experiment is shown in the following Table 1.

Table 1. Layout of the experiment

\begin{tabular}{ccccc}
\hline \multirow{2}{*}{$\begin{array}{c}\text { Dietary } \\
\text { Treatment }\end{array}$} & \multicolumn{3}{c}{ No of chicks } & $\begin{array}{c}\text { Total number } \\
\text { of chicks }\end{array}$ \\
\cline { 2 - 5 } $\mathbf{R}_{\mathbf{1}}$ & $\mathbf{R}_{\mathbf{2}}$ & $\mathbf{R}_{\mathbf{3}}$ & 39 \\
$\mathrm{~T}_{0} \mathrm{~F}$ & 13 & 13 & 13 & 39 \\
$\mathrm{~T}_{0} \mathrm{~A}$ & 13 & 13 & 13 & 39 \\
$\mathrm{~T}_{0} \mathrm{C}$ & 13 & 13 & 13 & 39 \\
$\mathrm{~T}_{0} \mathrm{H}$ & 13 & 13 & 13 & 39 \\
$\mathrm{~T}_{1} \mathrm{~F}$ & 13 & 13 & 13 & 39 \\
$\mathrm{~T}_{1} \mathrm{~A}$ & 13 & 13 & 13 & 39 \\
$\mathrm{~T}_{1} \mathrm{C}$ & 13 & 13 & 13 & 39 \\
$\mathrm{~T}_{1} \mathrm{H}$ & 13 & 13 & 13 & \\
\hline
\end{tabular}

${ }^{\#} T_{0} F$ : Fast feather feed on diet without phytase enzyme. $\mathrm{T}_{0} \mathrm{~A}$ : Arbor acres + giving diet without phytase enzyme. $\mathrm{T}_{0} \mathrm{C}$ : Coob-500 + giving diet without phytase enzyme. $\mathrm{T}_{0} \mathrm{H}$ : Hubbard classic + giving diet without phytase enzyme. $T_{1} \mathrm{~F}$ : Fast feather + giving diet having $1 \mathrm{~g}$ phytase enzyme/kg feed. $T_{1} A$ : Arbor acres + giving diet having $1 \mathrm{~g}$ phytase enzyme/kg feed. $\mathrm{T}_{1} \mathrm{C}$ : Cobb$500+$ giving diet having $1 \mathrm{~g}$ phytase enzyme $/ \mathrm{kg}$ feed. $\mathrm{T}_{1} \mathrm{H}$ : Hubbard classic + giving diet having $1 \mathrm{~g}$ phytase enzyme/kg feed.

\section{Preparation of the experimental house}

The experimental house was properly washed and cleaned using tap water. Ceiling, walls and floor were thoroughly cleaned and subsequently disinfected with Timsen $(1 \mathrm{gm} / \mathrm{lit})$. After proper drying, the house was divided into 24 pens of equal size using wire net. Each of pens was 12 sq feet floor spaces for 13 chicks. Fresh dried rice husk was used as litter material on the floor at a depth of 4 inch. 


\section{Experimental ration}

\section{Collection of enzyme}

The phytase enzyme (SQZYME SSF) (manufactured by F. Hoffmann-La Roche Ltd, Switzerland and supplied by Square limited, Bangladesh) was bought from the market for conducting the research work.

\section{Experimental diet}

Broiler starter and finisher diets were provided between 0 to 14 days and 15 to 35 days of age respectively. The diet was formulated using locally available feed ingredients such as Maize, Rice polish (Auto), Soybean meal (44), Protein Concentrate, Oyster shell, DCP, Soybean oil, Common salt, Vitamin-mineral premix (Rena premise). Coccidiostat (DOTT) added to the experimental diet as per recommendation of the manufacturer and mixed properly. Feed was prepared by mixing premix, enzyme and DOTT (Coccidiostat) firstly with a small quantity of mixed feed and then with gradually increased in quantity by adding remaining mixed feed. Finally, total amount of feed was mixed thoroughly. The whole procedure was followed for both starter and finisher diets. The amount of ingredients used and the detail composition of different experimental ration are shown in Table 2 .

Management of the experimental birds

At the beginning of the experiment, chicks were individually weighted and recorded as initial body weight. The following management procedures were followed during the experimental period.

\section{Litter management}

The birds were reared on rice husk litter floor having a depth of 4 inch. At the end of each week, litter was stirred to break its compactness and maintain proper moisture. At the end of the 4 weeks of age, droppings were cleaned from the surface of the litter.

\section{Brooding}

Additional heat was provided for brooding the chicks when it was necessary up to the end of $1^{\text {st }}$ week. Brooding temperature was maintained as bird's requirement from $33^{\circ} \mathrm{C}$ to normal environmental temperature of the house. Additional heat was managed by fitting 100 watt electric bulbs at the center of the pen, about 6 inches above of the floor. The heights of the bulbs were increased by raising the bulb gradually as per temperature requirement.

Table 2. Composition of the experimental starter and finished diets for broilers

\begin{tabular}{lcc}
\hline \multirow{2}{*}{ Feed ingredients } & \multicolumn{2}{c}{ Amount (kg/100kg feed) } \\
\cline { 2 - 3 } & $\begin{array}{c}\text { Starter (0- } \\
\text { 14 days) }\end{array}$ & $\begin{array}{c}\text { Finisher (15- } \\
\mathbf{3 5} \text { days) }\end{array}$ \\
\hline Maize & 53.5 & 57.00 \\
Rice polish (Auto) & 10.0 & 10.0 \\
Soybean meal (44) & 23.0 & 18.0 \\
Protein concentrate & 10.0 & 10.0 \\
Oyster shell & 1.0 & 0.75 \\
DCP & 0.5 & 0.75 \\
Soybean oil & 1.5 & 3.0 \\
Common salt & 0.25 & 0.25 \\
**Vitamin- mineral & 0.25 & 0.25 \\
premix & $\mathbf{1 0 0 k g}$ & $\mathbf{1 0 0 k g}$ \\
\hline \multicolumn{1}{c}{ Total } & Calculated composition \\
\cline { 2 - 3 }$\quad$ Starter (0- & Finisher (15- \\
\multicolumn{1}{c}{ Nutrients } & $\mathbf{1 4}$ days) & $\mathbf{3 5}$ days) \\
\hline ME (kcal/kg) & 2977 & 3074 \\
CP (\%) & 21.21 & 19.40 \\
CF (\%) & 5 & 5 \\
Ca (\%) & 1.00 & 0.95 \\
Available P (\%) & 0.74 & 0.75 \\
Ash (\%) & 6 & 6 \\
Lysine (\%) & 1.02 & 0.89 \\
Methionine (\%) & 0.35 & 0.35 \\
\hline
\end{tabular}

Note: Phytase enzyme used in enzyme group diet @ 10g/100kg.

**Vitamin-mineral premix composition (each $2.5 \mathrm{~kg}$ contained): Vitamin A 12000000 IU, Vitamin $D_{3} 2000000$ IU, Vitamin E $15000 \mathrm{mg}$, Vitamin $B_{1} 100 \mathrm{mg}$, Vitamin $B_{2} 4000 \mathrm{mg}$, Vitamin $B_{6} 3000$ mg, Vitamin $B_{12} 10 \mathrm{mg}$, Vitamin $\mathrm{K}_{3} 201 \mathrm{~K}$ ) mg, Folic acid $1500 \mathrm{mg}$, Nicotinic acid $25000 \mathrm{mg}$, Pantothenic acid $11000 \mathrm{mg}$, Biotin 15mg, Iron $32000 \mathrm{mg}$, Copper $8000 \mathrm{mg}$, Manganese $64000 \mathrm{mg}$, Cobalt $300 \mathrm{mg}$, Zinc $40000 \mathrm{mg}$, Iodine $800 \mathrm{mg}$, Selenium 200mg, Lysine $30000 \mathrm{mg}$, Methionine $50000 \mathrm{mg}$, Antioxidant $10000 \mathrm{mg}$. 


\section{Phytase for commercial broiler production}

\section{Lighting}

The birds were exposed to a continuous lighting of 23 hours and 30 minutes and a dark period of 30 minutes in 24 hours. Supplementary light at night was provided using electric bulb by hanging at a height of 2.8 meters to provide necessary lighting.

\section{Floor, feederand waterer space}

Each pen allotted for 13 birds, therefore floor space for each pen was $12 \mathrm{sq} \mathrm{ft}$. One round feeder and one round waterer provided in each pen for 13 birds, additional feeder and waterer added according to size of the flock.

\section{Feed and water management}

The birds were reared in separate pens. Feed and water was given ad-libitum throughout the experimental period.

\section{Vaccination and medication schedule}

The experimental birds were vaccinated against Baby chick Ranikhet Disease and Gumboro Disease. An antibiotic named Docolis $(0.5 \mathrm{~g} /$ liter of drinking water) used for first 3 days to prevent early chick mortality. Eskavit WS $(0.2 \mathrm{~g} /$ liter drinking water $)$, Electrocare $+(0.5 \mathrm{~g} /$ liter of drinking water $)$ were given to prevent strives.

\section{Data collection and record keeping}

Weekly body weight gain, feed consumption, feed conversion ration and cost benefit ration were recorded and statistically analyzed for a period of 5 weeks.

\section{Statistical analysis}

All recorded and calculated data were statistically analyzed by using analysis of variance (ANOVA) technique by a computer using "MSTAT statistical software" in accordance with the principles of Randomized Complete Block Design (RCBD). Duncan's Multiple Range Test was done to know the differences among the treatment means at $5 \%$ and $1 \%$ level of significance (Duncan, 1955)

\section{Results and Discussion}

\section{Effect of Phytase enzyme on body weight gain}

Tables 3 and 4 showed the growth performance affected by phytase enzyme supplementation during the different weekly intervals and the entire experimental period ( $2^{\text {nd }}$ to $5^{\text {th }}$ wks of age). The initial (1st week) body weight in all treatment groups were similar. Significant differences $(P>0.05)$ were found on body weight among the different treatment groups at $5^{\text {th }}$ weeks of age. The body weight of $T_{1} F$ was the highest at $2^{\text {nd }}$ weeks of age. The body weight of $\mathrm{T}_{0} \mathrm{~F}$ was higher than those of $\mathrm{T}_{1} \mathrm{~A}, \mathrm{~T}_{1} \mathrm{H}, \mathrm{T}_{1} \mathrm{C}$ at $2^{\text {nd }} \& 3^{\text {rd }}$ weeks of age. The highest body weight and body weight gain was attained by the birds under $\mathrm{T}_{1} \mathrm{C}$ at $5^{\text {th }}$ weeks of age. Among the treatment groups significant differences $(P<0.01)$ were found for broilers on $\mathrm{T}_{1} \mathrm{H}$ in comparison with $\mathrm{T}_{1} \mathrm{C}, \mathrm{T}_{1} \mathrm{~F}$ and $\mathrm{T}_{1} \mathrm{~A}$. All the strain of treatment groups showed better body weight than that of control groups. The highest body weight was found in $\mathrm{T}_{1} \mathrm{C}(1705.07 \mathrm{~g})$ and the lowest in $\mathrm{T}_{1} \mathrm{H}$ (1589.55). The result was consistent with the findings of Qian et al. (1996); Sebastian et al., (1996) and Nadeem et al. (2005). They reported that body weight and body weight gain were increased in chicks due to feeding diet containing phytase and utilization of phosphorus from the phytase mineral complex.

\section{Effect of Phytase enzyme on feed intake}

Table 4 and 5 represents the effect of phytase enzyme on feed intake of broilers. The differences in feed intake between control groups and treatment groups were significant $(P>0.05)$ at $5^{\text {th }}$ weeks of age. The result on feed intake demonstrated that during 28-35 days of age the birds in $T_{0} F$ consumed more feed than $T_{1} F, T_{0} C$, $T_{1} C, T_{0} A, T_{1} A, T_{0} H$ and $T_{1} H$ consequently. Among the treatment groups significant differences $(P<0.05)$ were found at $T_{1} \mathrm{H}$ in comparison with $T_{1} F, T_{1} C$ and $T_{1} A$. All the strains of treatment groups consumed less feed than that of control groups. The highest feed intake was found in $T_{1} F$ (2940.48g/bird) and the lowest in $T_{1} H$ (2638.47g/bird) among the treatment group. The result was consistent with the findings of some 
earlier workers (Wilson et al., 1999). They found that feed consumption was decreased due to addition of enzyme since birds fulfilled their nutrient requirement by taking less amount of feed. But this result was inconsistent with Ahmad et al., (2000) who reported that addition of phytase did not affect feed consumption.

Table: 3. Effect of Phytase enzyme on body weight of broilers ( $\mathrm{g} / \mathrm{bird}$ )

\begin{tabular}{|c|c|c|c|c|c|c|c|c|c|}
\hline \multirow{2}{*}{$\begin{array}{c}\text { Age } \\
\text { (Week) }\end{array}$} & \multicolumn{8}{|c|}{ Dietary treatment groups } & \multirow{2}{*}{$\begin{array}{l}\text { Level of } \\
\text { significance }\end{array}$} \\
\hline & $\mathrm{T}_{0} \mathrm{~F}$ & $\mathrm{~T}_{0} \mathrm{~A}$ & $\mathrm{~T}_{0} \mathrm{C}$ & $\mathrm{T}_{0} \mathrm{H}$ & $\mathrm{T}_{1} \mathrm{~F}$ & $\mathrm{~T}_{1} \mathrm{~A}$ & $\mathrm{~T}_{1} \mathrm{C}$ & $\mathrm{T}_{1} \mathrm{H}$ & \\
\hline \multirow{2}{*}{$\begin{array}{l}\text { Initial } \\
\text { weight }\end{array}$} & $41.67 \pm$ & 40.17 & 39.83 & 37.50 & 41.67 & \multirow{2}{*}{$\underset{0.49}{40.83} \pm$} & 40.0 & \multirow{2}{*}{$\underset{0.27}{39.67} \pm$} & \multirow[t]{2}{*}{ NS } \\
\hline & 01 & \pm 0.13 & \pm 0.13 & \pm 0.23 & \pm 0.13 & & \pm 0.47 & & \\
\hline \multirow[t]{2}{*}{$1^{\text {st }}$} & 151.8 & $132.3 \pm$ & $137.5 \pm$ & 125.4 & 151.7 & \multirow{2}{*}{$\begin{array}{c}133.2 \pm \\
1.54\end{array}$} & \multirow{2}{*}{$\begin{array}{c}139.9 \pm \\
1.19\end{array}$} & 127.5 & \multirow[t]{2}{*}{ NS } \\
\hline & \pm 3.01 & 0.90 & 1.75 & \pm 2.26 & \pm 2.74 & & & \pm 1.73 & \\
\hline \multirow[t]{2}{*}{$2^{\text {nd }}$} & 339.2 & $300.9 \pm$ & $327.5 \pm$ & 293.9 & 353.6 & \multirow{2}{*}{$\begin{array}{l}313.4 \pm \\
4.76^{b c d}\end{array}$} & \multirow{2}{*}{$\begin{array}{c}334.2 \pm \\
1.73^{a b c}\end{array}$} & \multirow{2}{*}{$\begin{array}{l}309.1 \pm \\
3.14^{c d}\end{array}$} & \multirow[t]{2}{*}{$* *$} \\
\hline & $\pm 6.18^{a b}$ & $5.21^{d}$ & $2.36^{a b c}$ & $\pm 2.55^{\mathrm{d}}$ & $\pm 5.30^{\mathrm{a}}$ & & & & \\
\hline \multirow[t]{2}{*}{$3^{\text {rd }}$} & 690.2 & 586.7 & $605.2 \pm$ & 604.8 & 746.1 & \multirow{2}{*}{$\begin{array}{c}637.7 \pm \\
5.19^{c}\end{array}$} & \multirow{2}{*}{$\begin{array}{c}633.5 \pm \\
5.66^{c}\end{array}$} & \multirow{2}{*}{$\begin{array}{r}637.1 \pm \\
3.01^{c}\end{array}$} & \multirow[t]{2}{*}{$* *$} \\
\hline & $\pm 1.43^{b}$ & $\pm 3.4^{\mathrm{d}}$ & $3.05^{d}$ & $\pm 4.26^{\mathrm{d}}$ & $\pm 10.66^{\mathrm{a}}$ & & & & \\
\hline \multirow[t]{2}{*}{$4^{\text {th }}$} & 1196.35 & $1065.43 \pm$ & 1173.04 & $1017.17 \pm$ & 1317.38 & \multirow{2}{*}{$\begin{array}{l}1176.41 \\
\pm 5.21^{b}\end{array}$} & 1230.00 & \multirow{2}{*}{$\begin{array}{l}1087.00 \\
\pm 3.38^{c}\end{array}$} & \multirow[t]{2}{*}{$* *$} \\
\hline & $\pm 8.66^{b}$ & $5.24^{c d}$ & $\pm 1.87^{b}$ & $3.00^{d}$ & $\pm 14.42^{a}$ & & $\pm 22.67^{b}$ & & \\
\hline \multirow[t]{2}{*}{$5^{\text {th }}$} & 1650.0 & 1526.10 & 1624.25 & 1468.06 & 1702.49 & \multirow{2}{*}{$\begin{array}{l}1681.54 \\
\pm 3.95^{a} \\
\end{array}$} & 1705.07 & \multirow{2}{*}{$\begin{array}{l}1589.55 \\
\pm 2.05^{d} \\
\end{array}$} & \multirow[t]{2}{*}{$* *$} \\
\hline & $\pm 0.13^{b c}$ & $\pm 9.28^{e}$ & $\pm 10.36^{c d}$ & $\pm 1.86^{\mathrm{f}}$ & $\pm 8.32^{\mathrm{a}}$ & & $\pm 8.70^{\mathrm{a}}$ & & \\
\hline
\end{tabular}

Values of different variables under different program indicates Mean \pm SEM; abcdefg mean values with dissimilar super scripts are significantly different; SEM , Standards errors of means ; ** Significant $(P<0.01)$; * Significant $(\mathrm{P}>0.05)$; NS (Non-significant). $\mathrm{T}_{0} \mathrm{~F}$ : Fast feather + giving diet without phytase enzyme. $T_{0} A$ : Arbor acres + giving diet without phytase enzyme. $\mathrm{T}_{0} \mathrm{C}$ : Coob-500 + giving diet without phytase enzyme. $\mathrm{T}_{0} \mathrm{H}$ : Hubbard classic + giving diet without phytase enzyme. $\mathrm{T}_{1} \mathrm{~F}$ : Fast feather + giving diet having $1 \mathrm{~g}$ phytase enzyme/kg feed. $\mathrm{T}_{1} \mathrm{~A}$ : Arbor acres + giving diet having $1 \mathrm{~g}$ phytase enzyme/kg feed. $\mathrm{T}_{1} \mathrm{C}$ : Cobb-500 + giving diet having $1 \mathrm{~g}$ phytase enzyme/kg feed. $\mathrm{T}_{1} \mathrm{H}$ : Hubbard classic + giving diet having $1 \mathrm{~g}$ phytase enzyme/kg feed.

Table: 4. Effect of Phytase enzyme on the production performance of broiler

\begin{tabular}{|c|c|c|c|c|c|c|c|c|c|c|}
\hline \multirow[t]{2}{*}{ Parameters } & \multirow{2}{*}{$\begin{array}{l}\text { Age in } \\
\text { (Week) }\end{array}$} & \multicolumn{8}{|c|}{ Dietary treatment groups } & \multirow{2}{*}{$\begin{array}{l}\text { Level of } \\
\text { significance }\end{array}$} \\
\hline & & $\mathrm{T}_{0} \mathrm{~F}$ & $\mathrm{~T}_{0} \mathrm{~A}$ & $\mathrm{~T}_{0} \mathrm{C}$ & $\mathrm{T}_{0} \mathrm{H}$ & $\mathrm{T}_{1} \mathrm{~F}$ & $\mathrm{~T}_{1} \mathrm{~A}$ & $\mathrm{~T}_{1} \mathrm{C}$ & $\mathrm{T}_{1} \mathrm{H}$ & \\
\hline $\begin{array}{l}\text { Body weight } \\
\text { (g/bird) }\end{array}$ & $0-5^{\text {th }}$ & $\begin{array}{l}1650.0 \\
\pm 0.13^{\mathrm{bc}}\end{array}$ & $\begin{array}{l}1526.10 \\
\pm 9.28^{\mathrm{e}}\end{array}$ & $\begin{array}{c}1624.25 \\
\pm \\
10.36^{\mathrm{cd}}\end{array}$ & $\begin{array}{l}1468.06 \\
\pm 1.86^{\mathrm{f}}\end{array}$ & $\begin{array}{l}1702.49 \\
\pm 8.32^{\mathrm{a}}\end{array}$ & $\begin{array}{l}1681.54 \\
\pm 3.95^{\mathrm{a}}\end{array}$ & $\begin{array}{r}1705.076 \\
\pm 8.70^{\mathrm{a}}\end{array}$ & $\begin{array}{l}1589.55 \\
\pm 2.05^{\mathrm{d}}\end{array}$ & $* *$ \\
\hline $\begin{array}{l}\text { Body weight gain } \\
\text { (g/bird) }\end{array}$ & $0-5^{\text {th }}$ & $\begin{array}{l}1608.31 \\
\pm 12.84^{\mathrm{abc}}\end{array}$ & $\begin{array}{l}1485.93 \\
\pm 9.30^{\mathrm{d}}\end{array}$ & $\begin{array}{c}1584.41 \\
\pm \\
10.24^{\mathrm{bc}}\end{array}$ & $\begin{array}{l}1428.06 \\
\pm 1.66^{\mathrm{e}}\end{array}$ & $\begin{array}{l}1661.72 \\
\pm 8.20^{\mathrm{a}}\end{array}$ & $\begin{array}{l}1640.70 \\
\pm 4.22^{\mathrm{ab}}\end{array}$ & $\begin{array}{l}1665.28 \\
\pm 8.87^{\mathrm{a}}\end{array}$ & $\begin{array}{l}1549.88 \\
\pm 1.96^{\mathrm{cd}}\end{array}$ & $* *$ \\
\hline $\begin{array}{l}\text { Feed consumption } \\
\text { (g/bird) }\end{array}$ & $0-5^{\text {th }}$ & $\begin{array}{l}3029.205 \\
\pm 12.84^{\mathrm{abc}}\end{array}$ & $\begin{array}{l}2788.042 \\
\pm 18.79^{\mathrm{c}}\end{array}$ & $\begin{array}{c}2883.48 \\
\pm \\
16.70^{\mathrm{bc}}\end{array}$ & $\begin{array}{l}2656.12 \\
\pm 20.20^{\mathrm{d}}\end{array}$ & $\begin{array}{c}2940.48 \\
\pm \\
8.20^{\mathrm{ab}}\end{array}$ & $\begin{array}{l}2826.78 \\
\pm 4.22^{\mathrm{ab}}\end{array}$ & $\begin{array}{l}2914.42 \\
\pm 8.70^{\mathrm{ab}}\end{array}$ & $\begin{array}{l}2638.47 \\
\pm 14.03^{\mathrm{d}}\end{array}$ & $*$ \\
\hline $\begin{array}{l}\text { Feed conversion } \\
\text { ratio }\end{array}$ & $0-5^{\text {th }}$ & $\begin{array}{l}1.88 \\
\pm 0.01^{\mathrm{a}}\end{array}$ & $\begin{array}{l}1.87 \\
\pm 0.02^{\mathrm{a}}\end{array}$ & $\begin{array}{l}1.82 \\
\pm 0.00^{\mathrm{ab}}\end{array}$ & $\begin{array}{l}1.86 \\
\pm 0.01^{\mathrm{a}}\end{array}$ & $\begin{array}{c}1.77 \\
\pm \\
0.01^{\mathrm{bc}}\end{array}$ & $\begin{array}{l}1.72 \\
\pm 0.01^{\mathrm{c}}\end{array}$ & $\begin{array}{l}1.75 \\
\pm 0.01^{\mathrm{bc}}\end{array}$ & $\begin{array}{c}1.70 \\
\pm 7.20^{\mathrm{c}}\end{array}$ & $* *$ \\
\hline $\begin{array}{l}\text { Profit / } \\
\text { broiler (Tk.) }\end{array}$ & $0-5^{\text {th }}$ & 25.09 & 20.64 & 33.88 & 14.5 & 35.43 & 37.87 & 36.96 & 34.69 & \\
\hline
\end{tabular}

Values of different variables under different program indicates Mean \pm SEM ; abcdefg mean values with dissimilar super scripts are significantly different; SEM, Standards errors of means; ** Significant $(P<0.01)$; * Significant $(P>0.05)$; NS (Non-significant). ToF: Fast feather + giving diet without phytase enzyme. $T_{0} A$ : Arbor acres + giving diet without phytase enzyme. $T_{0} C$ : Coob-500 + giving diet 
without phytase enzyme. $\mathrm{T}_{0} \mathrm{H}$ : Hubbard classic + giving diet without phytase enzyme. $\mathrm{T}_{1} \mathrm{~F}$ : Fast feather + giving diet having $1 \mathrm{~g}$ phytase enzyme/kg feed. $\mathrm{T}_{1} \mathrm{~A}$ : Arbor acres + giving diet having $1 \mathrm{~g}$ phytase enzyme/kg feed. $\mathrm{T}_{1} \mathrm{C}$ : Cobb-500 + giving diet having $1 \mathrm{~g}$ phytase enzyme/kg feed. $\mathrm{T}_{1} \mathrm{H}$ : Hubbard classic + giving diet having $1 \mathrm{~g}$ phytase enzyme/kg feed.

Table 5. Effect of Phytase enzyme in the diet of broilers on feed intake ( $\mathrm{g} / \mathrm{bird}$ )

\begin{tabular}{lcccccccccc}
\hline $\begin{array}{c}\text { Age } \\
\text { (Week) }\end{array}$ & \multicolumn{7}{c}{ Dietary treatment groups } \\
\cline { 2 - 10 } & $\mathrm{T}_{0} \mathrm{~F}$ & $\mathrm{~T}_{0} \mathrm{~A}$ & $\mathrm{~T}_{0} \mathrm{C}$ & $\mathrm{T}_{0} \mathrm{H}$ & $\mathrm{T}_{1} \mathrm{~F}$ & $\mathrm{~T}_{1} \mathrm{~A}$ & $\mathrm{~T}_{1} \mathrm{C}$ & $\mathrm{T}_{1} \mathrm{H}$ & $\begin{array}{c}\text { Level of } \\
\text { significance }\end{array}$ \\
\hline $1^{\text {st }}$ & 151.7 & 129.2 & 140.3 & 122.8 & 150.4 & 129.0 & 141.9 & 122.5 & $\mathrm{NS}$ \\
& \pm 5.28 & \pm 1.61 & \pm 2.00 & \pm 2.85 & \pm 3.71 & \pm 1.94 & \pm 2.46 & \pm 3.38 & \\
$2^{\text {nd }}$ & 460.8 & 399.0 & 437.2 & 397.7 & 445.1 & 394.2 & 442.4 & 388.7 & $\mathrm{NS}$ \\
& \pm 5.00 & \pm 10.71 & \pm 4.29 & \pm 2.35 & \pm 9.77 & \pm 6.78 & \pm 6.95 & \pm 1.97 & \\
$3^{\text {rd }}$ & 1076.40 & 896.8 & 915.8 & 947.9 & 1066.11 & 887.3 & 919.7 & 911.6 & $*$ \\
& $\pm 8.50^{\mathrm{a}}$ & $\pm 10.19^{\mathrm{bc}}$ & $\pm 5.85^{\mathrm{bc}}$ & $\pm 9.79^{\mathrm{d}}$ & $\pm 18.18^{\mathrm{a}}$ & $\pm 5.76^{\mathrm{c}}$ & $\pm 3.59^{\mathrm{dc}}$ & $\pm 12.85^{\mathrm{dc}}$ & \\
$4^{\text {th }}$ & 2059.66 & 1808.42 & 1949.06 & 1743.44 & 2084.75 & 18.09 .17 & 1918.42 & 1713.41 & $*$ \\
& $\pm 4.29^{\mathrm{a}}$ & $\pm 15.521^{\mathrm{c}}$ & $\pm 7.76^{\mathrm{d}}$ & $\pm 6.02^{\mathrm{cd}}$ & $\pm 32.331^{\mathrm{a}}$ & $\pm 11.11^{\mathrm{c}}$ & $\pm 27.59^{\mathrm{b}}$ & $\pm 3.28^{\mathrm{d}}$ & \\
$5^{\text {th }}$ & 3029.205 & 2788.042 & 2883.48 & 2656.12 & 2940.48 & 2826.78 & 2914.42 & 2638.47 & & $*$ \\
& $\pm 33.8^{\mathrm{a}}$ & $\pm 18.79^{\mathrm{c}}$ & \pm & $\pm 20.20^{\mathrm{d}}$ & $\pm 8.20^{\mathrm{ab}}$ & $\pm 4.22^{\mathrm{ab}}$ & $\pm 8.70^{\mathrm{ab}}$ & $\pm 14.03^{\mathrm{d}}$ & $*$ \\
& & & $16.70^{\mathrm{bc}}$ & & & & & & \\
\hline
\end{tabular}

Values of different variables under different program indicates Mean \pm SEM ; abcdefg mean values with dissimilar super scripts are significantly different; SEM, Standards errors of means; ** Significant $(P<0.01)$; * Significant $(\mathrm{P}>0.05)$; NS (Non-significant). $\mathrm{T}_{0} \mathrm{~F}$ : Fast feather + giving diet without phytase enzyme. $\mathrm{T}_{0} \mathrm{~A}$ : Arbor acres + giving diet without phytase enzyme. $\mathrm{T}_{0} \mathrm{C}$ : Coob-500 + giving diet without phytase enzyme. $\mathrm{T}_{0} \mathrm{H}$ : Hubbard classic + giving diet without phytase enzyme. $\mathrm{T}_{1} \mathrm{~F}$ : Fast feather + giving diet having $1 \mathrm{~g}$ phytase enzyme/kg feed. $\mathrm{T}_{1} \mathrm{~A}$ : Arbor acres + giving diet having $1 \mathrm{~g}$ phytase enzyme/kg feed. $T_{1} \mathrm{C}$ : Cobb-500 + giving diet having $1 \mathrm{~g}$ phytase enzyme/kg feed. $\mathrm{T}_{1} \mathrm{H}$ : Hubbard classic + giving diet having $1 \mathrm{~g}$ phytase enzyme/kg feed.

\section{Effect of Phytase enzyme on feed conversion ratio (FCR) of broilers}

Table 4 and 6 showed that significant differences $(P>0.01)$ were found on feed conversion ratio at $5^{\text {th }}$ weeks of age. The feed conversion ratio between control groups and treatment groups were significantly different $(P<0.01)$. Results showed that among the four different strains, Hubbard classic had the lowest FCR, suggesting that this broiler strain could utilize the feed efficiently compared to other three strains. All the strain of treatment groups showed better FCR than control groups. At the end of trial, the feed conversion ratio of $T_{1} \mathrm{H}$ was the lowest followed by $T_{1} A, T_{1} C, T_{1} F, T_{0} C, T_{0} H, T_{0} A, T_{0} F$, respectively.
All the strains of treatment groups have significantly $(P>0.05)$ lower feed conversion ratio than that of control groups. The finding of the present study agreed with the results of some earlier studies (Selle et al., 2006; Watson et al., 2006). In those studies, feed conversion ratio of different broiler groups differed significantly might be due to the increased availability of energy and amino acid digestibility by the addition of phytase enzyme. The higher body weight gain and lower feed conversion ratio of chicks might be due to the increased availability of energy (Shirley and Edwards, 2003) and amino acid digestibility (Selle et al., 2006) due to raise in phytate degredation. The ability of phytase to improve Phosphorus availability by hydrolyzing phytate-bound $P$ in poultry diets can therefore reduce supplementation of diets with inorganic $P$ sources. 
Parvin et al. (2016) Bang. J. Anim. Sci. 45 (3): 31-39

Table 6. Effect of Phytase enzyme in the diet of broilers on feed conversion ratio

\begin{tabular}{|c|c|c|c|c|c|c|c|c|c|}
\hline \multirow{2}{*}{$\begin{array}{c}\text { Age } \\
\text { (Week) }\end{array}$} & \multicolumn{8}{|c|}{ Dietary treatment groups } & \multirow{2}{*}{$\begin{array}{l}\text { Level of } \\
\text { significance }\end{array}$} \\
\hline & $\mathrm{T}_{0} \mathrm{~F}$ & $\mathrm{~T}_{0} \mathrm{~A}$ & $\mathrm{~T}_{0} \mathrm{C}$ & $\mathrm{T}_{0} \mathrm{H}$ & $\mathrm{T}_{1} \mathrm{~F}$ & $\mathrm{~T}_{1} \mathrm{~A}$ & $\mathrm{~T}_{1} \mathrm{C}$ & $\mathrm{T}_{1} \mathrm{H}$ & \\
\hline $1^{\text {st }}$ & $\begin{array}{c}1.37 \\
\pm 0.01\end{array}$ & $\begin{array}{c}1.40 \\
\pm 0.00\end{array}$ & $\begin{array}{c}1.43 \\
\pm 0.01\end{array}$ & $\begin{array}{c}1.43 \\
\pm 0.09\end{array}$ & $\begin{array}{c}1.36 \\
\pm 0.07\end{array}$ & $\begin{array}{c}1.39 \\
\pm 0.07\end{array}$ & $\begin{array}{c}1.42 \\
\pm 0.12\end{array}$ & $\begin{array}{c}1.39 \\
\pm 0.01\end{array}$ & NS \\
\hline $2^{\text {nd }}$ & $\begin{array}{c}1.54 \\
\pm 0.01^{\mathrm{a}}\end{array}$ & $\begin{array}{c}1.52 \\
\pm 0.02^{\mathrm{ab}}\end{array}$ & $\begin{array}{c}1.51 \\
\pm 0.01^{\mathrm{ab}}\end{array}$ & $\begin{array}{c}1.55 \\
\pm 0.05^{\mathrm{a}}\end{array}$ & $\begin{array}{c}1.42 \\
\pm 0.04^{\mathrm{c}}\end{array}$ & $\begin{array}{c}1.44 \\
\pm 0.01^{\mathrm{bc}}\end{array}$ & $\begin{array}{c}1.48 \\
\pm 0.01^{\mathrm{abc}}\end{array}$ & $\begin{array}{c}1.43 \\
\pm 0.02^{\mathrm{bc}}\end{array}$ & $* *$ \\
\hline $3^{\text {rd }}$ & $\begin{array}{c}1.65 \\
\pm 0.01^{\mathrm{a}}\end{array}$ & $\begin{array}{c}1.63 \\
\pm 0.01^{\mathrm{a}}\end{array}$ & $\begin{array}{c}1.61 \\
\pm 0.01^{\mathrm{ab}}\end{array}$ & $\begin{array}{c}1.67 \\
\pm 0.09^{\mathrm{a}}\end{array}$ & $\begin{array}{c}1.50 \\
\pm 0.05^{\mathrm{c}}\end{array}$ & $\begin{array}{c}1.47 \\
\pm 0.07^{\mathrm{c}}\end{array}$ & $\begin{array}{c}1.54 \\
\pm 0.09^{\mathrm{bc}}\end{array}$ & $\begin{array}{c}1.51 \\
\pm 0.01^{\mathrm{c}}\end{array}$ & $* *$ \\
\hline $4^{\text {th }}$ & $\begin{array}{c}1.77 \\
\pm 0.01^{\mathrm{a}}\end{array}$ & $\begin{array}{c}1.75 \\
\pm 0.02^{\mathrm{a}}\end{array}$ & $\begin{array}{c}1.71 \\
\pm 0.09^{\mathrm{a}}\end{array}$ & $\begin{array}{c}1.77 \\
\pm 0.09^{\mathrm{a}}\end{array}$ & $\begin{array}{c}1.62 \\
\pm 0.01^{\mathrm{b}}\end{array}$ & $\begin{array}{c}1.58 \\
\pm 0.01^{\mathrm{b}}\end{array}$ & $\begin{array}{c}1.60 \\
\pm 0.07^{\mathrm{b}}\end{array}$ & $\begin{array}{c}1.62 \\
\pm 0.07^{\mathrm{b}}\end{array}$ & $* *$ \\
\hline $5^{\text {th }}$ & $\begin{array}{c}1.88 \\
\pm 0.01^{\mathrm{a}}\end{array}$ & $\begin{array}{c}1.87 \\
\pm 0.02^{\mathrm{a}}\end{array}$ & $\begin{array}{c}1.82 \\
\pm 0.0^{\mathrm{a}}\end{array}$ & $\begin{array}{c}1.86 \\
\pm 0.01^{\mathrm{a}}\end{array}$ & $\begin{array}{c}1.77 \\
\pm 0.01^{\mathrm{b}}\end{array}$ & $\begin{array}{c}1.72 \\
\pm 0.01^{\mathrm{b}}\end{array}$ & $\begin{array}{c}1.75 \\
\pm 0.01^{\mathrm{b}}\end{array}$ & $\begin{array}{c}1.70 \\
\pm 0.07^{\mathrm{c}}\end{array}$ & $* *$ \\
\hline
\end{tabular}

Values of different variables under different program indicates Mean $\pm S E M$; abcdefg mean values with dissimilar super scripts are significantly different; SEM , Standards errors of means; ** Significant $(P<0.01)$; * Significant $(P>0.05)$; NS (Non-significant). Here, $\mathrm{T}_{0} \mathrm{~F}$ : Fast feather + giving diet without phytase enzyme. $\mathrm{T}_{0} \mathrm{~A}$ : Arbor acres + giving diet without phytase enzyme. $\mathrm{T}_{0} \mathrm{C}$ : Coob-500 + giving diet without phytase enzyme. $\mathrm{T}_{0} \mathrm{H}$ : Hubbard classic + giving diet without phytase enzyme. $\mathrm{T}_{1} \mathrm{~F}$ : Fast feather + giving diet having $1 \mathrm{~g}$ phytase enzyme/kg feed. $\mathrm{T}_{1} \mathrm{~A}$ : Arbor acres + giving diet having $1 \mathrm{~g}$ phytase enzyme/kg feed. $\mathrm{T}_{1} \mathrm{C}$ : Cobb-500 + giving diet having $1 \mathrm{~g}$ phytase enzyme/kg feed. $\mathrm{T}_{1} \mathrm{H}$ : Hubbard classic + giving diet having $1 \mathrm{~g}$ phytase enzyme/kg feed.

\section{Effect of Phytase enzyme on production cost and profit margin of broilers}

The production cost of broiler was shown in Table 7. The feed cost was the highest (Tk. 5316) in treatment $T_{0} F$ and the lowest (Tk. 4630) in treatment $\mathrm{T} 1 \mathrm{H}$ group. The total cost Taka per $\mathrm{kg}$ live weight of broiler was the highest in treatment $T_{1} F$ (180.15) followed by treatment $T_{1} C$ (179.0), $\mathrm{T}_{0} \mathrm{~F}$ (178.41), $\mathrm{T}_{1} \mathrm{~A}$ (175.05), $\mathrm{T}_{0} \mathrm{C}$ (171.82), $\mathrm{T}_{0} \mathrm{~A}$ (167.56), $\quad \mathrm{T}_{1} \mathrm{H} \quad(166.58) \quad \& \quad \mathrm{~T}_{0} \mathrm{H} \quad$ (161.61) respectively. Net profit per live broiler was the highest in treatment $T_{1} A$ (Tk. 37.87) followed by treatment $\mathrm{T}_{1} \mathrm{C}$ (36.96), $\mathrm{T}_{1} \mathrm{~F}$ (35.43), $\mathrm{T}_{1} \mathrm{H}$ (34.69), $\mathrm{T}_{0} \mathrm{C}$ (33.88), $\mathrm{T}_{0} \mathrm{~F}$ (25.09), $\mathrm{T}_{0} \mathrm{~A}$ (20.64) and $\mathrm{T}_{0} \mathrm{H}$ (14.55) respectively. The highest net profit was observed in treatment $\mathrm{T}_{1} \mathrm{~A}$ and the lowest total net profit was observed in treatment $\mathrm{T}_{0} \mathrm{H}$. Economic wise, the addition of phytase could make reasonable profits than without its addition. The findings of the present study is similar to the observation of Islam et al. (2010).
They reported that net profits (Kg live bird) was significantly better in broiler group fed diet supplemented with enzyme $50 \mathrm{~g} / 100 \mathrm{~kg}$ which might be due to improvement of digestibility's and consequent by better utilization of nutrients. Vinil et al. (2000) found a reduction in feed cost in soy-wheat bran diets supplemented with phytase (25 g $100 \mathrm{~kg}-1$ ) of about 1.00 Indian rupee (INR). Net income increased up to $9.47 \%$ in response to $300 \mathrm{~g} / \mathrm{ton}$ of phytase supplementa-tion (Kundu et al. 2000). Singh and Khatta (2004) reported that phytase supplementation resulted in $10 \%$ and $6 \%$ reductions in cost per unit gain in broilers fed corn and wheat based diets, respectively. Plumstead et al. (2008) observed that less expensive broiler diets low in $\mathrm{P}$ and other nutrients supplemented with phytase resulted in optimum production. Supplementation of phytase leads to safe, economic and almost complete replacement of dietary $P$ (dicalcium phos-phate), that ultimately causes reduction in feed cost $\mathrm{kg}-1$ of weight gain (Singh and Khatta, 2003). 
Table 7. Effect of Phytase enzyme on production cost and profit margin of broilers

\begin{tabular}{lcccccccc}
\hline \multicolumn{1}{c}{ Cost of production } & \multicolumn{7}{c}{ Dietary treatment groups } \\
\cline { 2 - 9 } & $\mathrm{T}_{0} \mathrm{~F}$ & $\mathrm{~T}_{0} \mathrm{~A}$ & $\mathrm{~T}_{0} \mathrm{C}$ & $\mathrm{T}_{0} \mathrm{H}$ & $\mathrm{T}_{1} \mathrm{~F}$ & $\mathrm{~T}_{1} \mathrm{~A}$ & $\mathrm{~T}_{1} \mathrm{C}$ & $\mathrm{T}_{1} \mathrm{H}$ \\
\hline Feed cost @45tk/kg & 5316 & 4893 & 5059 & 4661 & 5159 & 4960 & 5114 & 4630 \\
Chick cost @32tk/bird & 1248 & 1248 & 1248 & 1248 & 1248 & 1248 & 1248 & 1248 \\
Disinfectant cost & 10 & 10 & 10 & 10 & 10 & 10 & 10 & 10 \\
Vaccination cost & 50 & 50 & 50 & 50 & 50 & 50 & 50 & 50 \\
Medicine cost & 24 & 24 & 24 & 24 & 24 & 24 & 24 & 24 \\
Transport cost & 10 & 10 & 10 & 10 & 10 & 10 & 10 & 10 \\
Depreciation cost & 150 & 150 & 150 & 150 & 150 & 150 & 150 & 150 \\
Litter cost & 50 & 50 & 50 & 50 & 50 & 50 & 50 & 50 \\
Labour cost & 60 & 60 & 60 & 60 & 60 & 60 & 60 & 60 \\
Water and electricity & 40 & 40 & 40 & 40 & 40 & 40 & 40 & 40 \\
cost & & & & & 225 & 225 & 225 & 225 \\
Phytase enzyme cost & $\mathbf{6 9 5 8}$ & $\mathbf{6 5 3 5}$ & $\mathbf{6 7 0 1}$ & $\mathbf{6 3 0 3}$ & $\mathbf{7 0 2 6}$ & $\mathbf{6 8 2 7}$ & $\mathbf{6 9 8 1}$ & $\mathbf{6 4 9 7}$ \\
\hline Total Cost & 178.41 & 167.56 & 171.82 & 161.61 & 180.15 & 175.05 & 179 & 166.58 \\
\hline Cost/kg broiler & 7936.5 & 7340.06 & 8022.56 & 6870.24 & 8407.88 & 8304.14 & 8422.7 & 7849.66 \\
Total selling @ & 25.09 & 20.64 & 33.88 & 14.55 & 35.43 & 37.87 & 36.96 & 34.69 \\
130tk/kg & & & & & & & \\
Profit/ broiler & & & & & & & & \\
\hline
\end{tabular}

$\mathrm{T}_{0} \mathrm{~F}$ : Fast feather + giving diet without phytase enzyme. $\mathrm{T}_{0} \mathrm{~A}$ : Arbor acres + giving diet without phytase enzyme. $\mathrm{T}_{0} \mathrm{C}$ : Coob-500 + giving diet without phytase enzyme. $\mathrm{T}_{0} \mathrm{H}$ : Hubbard classic + giving diet without phytase enzyme. $\mathrm{T}_{1} \mathrm{~F}$ : Fast feather + giving diet having $1 \mathrm{~g}$ phytase enzyme/kg feed. $T_{1} A$ : Arbor acres + giving diet having $1 \mathrm{~g}$ phytase enzyme $/ \mathrm{kg}$ feed. $\mathrm{T}_{1} \mathrm{C}$ : Cobb$500+$ giving diet having $1 \mathrm{~g}$ phytase enzyme/kg feed. $\mathrm{T}_{1} \mathrm{H}$ : Hubbard classic + giving diet having $1 \mathrm{~g}$ phytase enzyme/kg feed.

\section{Conclusion}

The current results indicate that addition of phytase enzyme @ $1 \mathrm{~g} / \mathrm{kg}$ feed of broilers of different strains improved body weight gain lowering the feed efficiency due to better nutrient availability in comparison to strains of control groups. The cost of production per kg broiler was lower in phytase supplemented stains, which ultimately made the higher net profit per broiler

\section{References}

Ahmad T, S Rasool, M Sarwar, A Haq and Z Hasan (2000). Effect of microbial phytase produced from a fungus aspergillus niger on biovailability of phosphorus and calcium in broiler chickens. Anim. Feed Sci. and Technol., 83(2): 103-114.

Dilger RN, EM Onyango and JS Sands (2004). Evaluation of microbial phytase in broiler diets. Poult. Sci., 83:962-970.

Duncan DB (1955). Multiple Range and Multiple $F$ Tests. Biometrics, 11, pp.1-42.

Fukayama EH, NK Sakomura and LRB Dourado (2008). Effect of phytase suplementation on performance and nutrient digestibility in diets of broilers. R. Bras. Zootec., 37 (4): 629-635,

Islam FA, O Simon, G Milksch and K Manner (2010). Comparative studies of the in vitro properties of phytases from various microbial origins. Arch. Anim. Nutr. 53, 353-373.

Kundu B, P Biswas, D Rajendran and SK Dandopath (2000). Effect of supplementation of phytase enzyme and gradual re-placement of dicalcium phosphate on the performance of commercial broker chicken. PP: 119-128 in Proc. III Biennial conference of Animal Nutrition Association, Hisar, India.

Nadeem MA, MI Anjum, AG Khan and A Azim (2005). Effect of dietary supplementation of non- starch polysaccharide degrading enzymes on growth performance of broiler chicks. Pak. Vet. J. 25(4): 183-187.

NRC (1994). Nutrient requirements of poultry. 9th edn. National Academy Press, Washington, DC.

Plumstead PW, AB Leytem and RO Maguire (2008). Interac-tion of calcium and phytate in broiler diets. Effects on appar-ent prececal digestibility and retention of phosphorus. Poult. Sci. 87: 449-458.

Qian H, HP Vett, ET Kornegay, V Ravindran and DM Denbow (1996). Effects of supplemental phytase and phosphorus on histological and other tibial bone characteristics and performance of broilers fed semi-purified diets. Poult. Sci. 75 (5): 618-626.

Sebastian S, SP Touchburn, ER Chavez and PC Lague (1996). Apparent digestibility of protein and amino acids in broiler chickens fed a cornsoybean diet supplemented with microbial phytase. Poult. Sci. 76:1760-1769. 
Selle PH, V Ravindran, WL Bryden and T Scott (2006). Influence of dietary phytate and exogenous phytase on amino acid digestibility in poultry. J. Poult. Sci. 43:89-103.

Shirley R and HM Edwards Jr. (2003). Graded levels of phytase past industry standards improves broiler performance. Poult. Sci. 82: 671-680.

Singh PK and VK Khatta (2002). Phytase supplementation for economic and eco-friendly broiler production. J. Eco. Physiol. 5: 117-121.

Singh PK and VK Khatta (2003). Effect of phytase supple-mentation on the broiler production. Poult. Planner, 4: 13-14.

Singh PK and VK Khatta (2004). Economics of broilers raised on phytase supplemented diets. Ind. J. Anim. Res. 10: 121-124.
Vinil SP, C Kadirvelan, S Thirumalai, S Valli S and B Chandrabose (2000). The role of phytase and other enzymes in augment-ing the bioavailability of nutrients in broilers. Pp. 97 in Proc. xx Annual Conference of Indian Poultry Science Association, Chennai, India.

Watson BC, JO Matthews, LL Southern and JL Shelton (2006). "The effects of phytase on growth performance and intestinal transit time of broilers fed nutritionally adequate diets and diets deficient in calcium and phosphorus," Poult. Sci. 85(3): 493-497.

Wilson W, C Chicco, F Meschy and F Requena (1999). Phytic phosphorus and phytase activity in chickens. Interciencia, 30: 24-28. 